\title{
FINITE AND COUNTABLE ADDITIVITY OF TOPOLOGICAL PROPERTIES IN NICE SPACES
}

\author{
V. V. TKACHUK
}

\begin{abstract}
Let $Q \in$ character $\leq \tau$, pseudocharacter $\leq \tau$, tightness $\leq$ $\tau$, weight $\leq \tau, P_{\tau}$-property, discreteness, Fréchet-Urysohn property, sequentiality, radiality, pseudoradiality, local compactness, $k$-property $\}$. If $X^{n}=$ $\bigcup\left\{X_{i}: i \in n\right\}, X_{i} \vdash Q$ for all $i \in n$ then $X \vdash Q$ (i.e. the property $Q$ is $n$-additive in $X^{n}$ for any $\left.X \in T_{3}\right)$. Metrizability is $n$-additive in $X^{n}$ provided $X$ is compact or $c(X)=\omega$. ANR-property is closely $n$-additive in $X^{n}$ if $X$ is compact ("closely" means additivity in case $X_{i}$ is closed in $X^{n}$ ). If $Q \in$ \{metrizability, character $\leq \tau$, pseudocharacter $\leq \tau$, diagonal number $\leq \tau, i$-weight $\leq \tau$, pseudoweight $\leq \tau$, local compactness $\}$ then $Q$ is finitely additive in any topological group.
\end{abstract}

\section{INTRODUCTION}

There are quite a few properties finitely or countably additive in every space. The network weight and density are classical examples hereof. The weight and metrizability can serve as examples of nonadditive topological properties which are also numerous. However the weight is countably additive sometimes. This happens in metric as well as in compact spaces. We are going to show that nonadditive topological properties are actually additive in much more cases than could be seen at the first glance.

Let us start with definitions. Given a space $X$ and an $n \in \omega$ we say that a property $Q$ is (closely) $n$-additive in $X$ if $X \vdash Q(X$ has $Q)$ in case $X=X_{0} \cup \cdots \cup X_{n-1}$ where $X_{i} \vdash Q$ (and is closed in $X$ respectively) for all $i \in n$. A property $Q$ is countably additive in $X$ if $X \vdash Q$ as soon as $X=\bigcup\left\{X_{i}: i \in \omega\right\}$ and $X_{i} \vdash Q$ for $i \in \omega$.

Suppose that we are trying to find as large a class as possible in which most of nonadditive properties become additive. Can it be in some way the class of all regular spaces (or $T_{1}$-spaces)? The space $A\left(\omega_{1}\right)$ (which is the Alexandroff compactification of the discrete space of power $\left.\omega_{1}\right)$ seems to dispel all hopes of that, because it shows that a union of two metrizable spaces can have an uncountable pseudocharacter. It can also have an uncountable tightness and can be an example of nonadditivity for almost all nonadditive properties at once.

It turns out however that a slight change of generality results in additivity for all properties one could imagine. That is the following will be proved.

Received by the editors January 8, 1991 and, in revised form, July 26, 1991.

1980 Mathematics Subject Classification (1985 Revision). Primary 54A25. 
0 . Theorem. Let $Q$ be a topological property and $n \in \omega \backslash\{0\}$. Suppose that $Q$ belongs to some list of more than twenty properties and $X^{n}=\bigcup\left\{X_{i}: i \in n\right\}$, where $X_{i} \vdash Q$. Then $X \vdash Q$.

Of course, this list includes weight, character, pseudocharacter and most of other known nonadditive properties. In fact it is very difficult to find a property $Q$ for which the Theorem 0 does not hold. The only $Q$ the author succeeded to discover is "ind $\leq 1$ ". It seims curious that $Q=$ "ind $\leq k$ " is closely $n$-additive for any $X^{n}$ being not closely 2 -additive in the class of compact spaces. It is easy to prove that most nonadditive properties are not $(n+1)$-additive in $X^{n}$ even in case $X$ is compact.

The results on $n$-additivity of properties for $X^{n}$ make up $\S 1$ of this paper. In $\S 2$ we deal with countable adiditivity of properties for $X^{\omega}$. A. V. Arhangel'skii's problem is the main one here. He asked if metrizability is countably additive for $X^{\omega}$. M. G. Tkačenko proved in [1] that the answer is "yes" for all $X$ with $\mathrm{cl}^{*}(X) \leq \omega$. In particular, metrizability is countably additive in $X^{\omega}$ for separable as well as for ccuntably compact $X$. We prove that metrizability is finitely additive in $X^{\omega}$ for any $X$.

In $\S 3$ we prove that some properties and cardinal furictions are finitely additive in topological groups and some are not.

Notations and terminology. If $X$ is a space then $T(X)$ is its topology and $T^{*}(X)=T(X) \backslash\{0\}, T(x, X)=\{U \in T(X): x \in U\}$ for any $x \in X$. The expression $X \simeq Y$ means the spaces $X$ and $Y$ are homeomorphic. If $B$ is a family of subsets of $X$ and $A \subset X$ then $B \mid A=\{U \cap A: U \in B$, $U \cap A \neq 0\}$ and $B(x)=\{U \in B: U \ni x\}$. A point $x \in X$ is a $P_{\tau}$-point in $X$ iff $\bigcap\left\{U_{\alpha}: \alpha<\tau\right\}$ is a neighbourhood of $x$ as soon as $U_{\alpha} \in T(x, X)$ for $\alpha<\tau$. A space $X$ is a $P_{\tau}$-space if every $x \in X$ is a $P_{\tau}$-point in $X$. A set $H \subset X$ is called a $G_{\tau}$-set if $H=\bigcap\left\{U_{\alpha}: \alpha<\tau\right\}$ where $U_{\alpha} \in T(X), \alpha<\tau$. The symbols $\alpha, \beta$ always serve as ordinals, $\tau$ is for a cardinal, $F U$ abbreviates "FréchetUrysohn". If $X$ is a space and $A \subset X$ then $\bar{A}^{\tau}=\bigcup\{\bar{B}: B \subset A$ and $|B| \leq \tau\}$. If $A \subset Y \subset X$ then $\bar{A}^{Y}$ as well as $\operatorname{cl}_{Y}(A)$ is the closure of the set $A$ in $Y$. i he same bar is used to denote closures in different spaces if no confusion is possible. A space $X$ is a $k$-space if $A \subset X, \bar{A} \neq A$ implies $\overline{A \cap K} \neq A \cap K$ for some compact $K \subset X$. We say that $X$ is $\omega$-monolithic if $n w(\bar{A}) \leq \omega$ for every countable $A \subset X$. The space $L\left(\omega_{1}\right)$ is the lindelöfication of the discrete space of power $\omega_{1}$. That is $L\left(\omega_{1}\right)=L \cup\{a\}, L \not \supset a, L \subset T\left(L\left(\omega_{1}\right)\right)$, $T\left(a, L\left(\omega_{1}\right)\right)=\{\{a\} \cup(L \backslash A):|A| \leq \omega\}$. All other notions are standard.

\section{AdDITIVITY IN FINITE POWERS}

Most of our results on $n$-additivity in $X^{n}$ concern pointwise properties. A property $Q$ is called pointwise if for every space $X$ we have $X \vdash Q$ if and only if $X \vdash Q(x, X)$ for all $x \in X$. Here $Q(x, X)$ is some point property in $X$. A class $C$ of subspaces is said to be representative for the property $Q$ if for every space $X$ and any $H \subset X$ with $H \in C$ we have $X \vdash Q(x, H)$ iff $X \vdash Q(x, X)$ for any $x \in H$.

We are not going to give rigorous definitions of "point property" and "representative class". We will just define what $Q(x, X)$ and representative sets are for any particular $Q$. We will also be informal enough to say that a car- 
dinal function $\xi$ is $n$-additive in $X^{n}$. This will mean the property " $\xi \leq \tau$ " is $n$-additive in $X^{n}$ for any $\tau$.

1.1. Definition. Given a space $X$ and a property $Q$ let $Q_{\text {loc }}(x, X)=$ "there is a $U \in T(x, X)$ such that $\bar{U} \vdash Q$ ". A space $X$ is called locally $Q$-space iff $Q_{\text {loc }}(x, X)$ for every $x \in X$.

If $x \in X$, then $x^{n} \in X^{n}$ is the point with all its coordinates equal to $x$.

\subsection{Theorem. Suppose that the property $Q$ satisfies}

(i) if $X \vdash Q$ and $U \in T^{*}(X)$ then $\bar{U} \vdash Q$;

(ii) if $f: X \rightarrow Y$ is a continuous onto map and $X \vdash Q$, then $Y \vdash Q$. Then $Q_{\text {loc }}$ is $n$-additive in $X^{n}$ for all $n \geq 1$ and $X \in T_{3}$.

Proof. The property $Q_{\text {loc }}$ must not be invariant with respect to continuous maps. However it behaves differently where retractions come in.

1.3. Lemma. If $Q \vdash$ (i) $\wedge$ (ii), $X \vdash Q_{\mathrm{loc}}$ and $r: X \rightarrow Y$ is a retraction then $Y \vdash Q_{\text {loc }}$.

Proof of the lemma. Pick any $y \in Y$. There is a $U \in T(y, X)$ with $\bar{U} \vdash Q$. Let $V=U \cap Y$. Then $V \in T(y, Y)$ and $V \subset r(\bar{U})$. Pick any $W \in T(y, Y)$ with $\bar{W}^{Y} \subset V$. It follows from $r(\bar{U}) \vdash Q$ and (i) that $\bar{W}^{Y} \vdash Q$ and we established $Q_{\text {loc }}(y, Y)$ together with the Lemma 1.3.

Resuming the proof of 1.2 consider the following statement $I_{k}$ for $k \geq 1$. $I_{k}=$ "if $X^{k}$ is a retract of some $Y$ with $X_{i} \subset Y, X_{i} \vdash Q_{\text {loc }}(i \in k)$ and $\bigcup\left\{X_{i}: i \in k\right\} \supset X^{k}$ then $X \vdash Q_{\text {loc }}$. It suffices that we prove $I_{k}$ for all $k \geq 1$. We will do that using induction along $k$. If $k=1$ then $X$ is a retract of $X_{0}$ so that this situation was dealt with in Lemma 1.3.

Assume that $I_{k}$ is true for $k \leq n-1$. Let $X^{n}$ be a retract of $Y$ and $X^{n} \subset \bigcup\left\{X_{i}: i \in n\right\}$ where $X_{i} \vdash Q_{\text {loc }}$ for $i \in n$. Pick any $x \in X$ and $j \in n$ with $x^{n} \in X_{j}$. There exists a set $V \in T\left(x^{n}, Y\right)$ such that

$$
H=\operatorname{cl}_{X_{j}}\left(V \cap X_{j}\right) \vdash Q .
$$

The set $V \cap X^{n}$ contains $U^{n}$ for some $U \in T(x, X)$. Denote by $r$ the retraction from $Y$ onto $X^{n}$. The set $W=r^{-1}\left(U^{n}\right)$ is open in $Y$ so that $Y_{i}=X_{i} \cap W \vdash Q_{\mathrm{loc}}$ for $i \in n$. Clearly, $U^{n} \subset W$ and $r(W)=U^{n}$ i.e. $U^{n}$ is a retract of $W$. Suppose that there is a point $y \in U$ with $\left(\{y\} \times U^{n-1}\right) \cap X_{j}=0$. Then $\{y\} \times U^{n-1}$ is homeomorphic to $U^{n-1}$ and being a retract of $W$ is covered by $\leq n-1$ subsets of $W$ all of which have $Q_{\text {loc }}$. The induction hypothesis yields $U \vdash Q_{\text {loc }}$ and $Q_{\text {loc }}(x, X)$ and that is what we needed.

If $\left(\{y\} \times U^{n-1}\right) \cap X_{j} \neq 0$ for all $y \in U$ then $U \subset p_{1}\left(X_{j} \cap U^{n}\right)$, where $p_{1}: X^{n} \rightarrow X$ is the natural projection of $X^{n}$ onto its first factor. But $X_{j} \cap U^{n} \subset$ $r(H)$ so that $U \subset p_{1}(r(H))$. The set $G=p_{1}(r(H))$ has $Q$ by (ii). Now it suffices to pick any $U_{1} \subset U, U_{1} \in T(x, X)$ with $\bar{U}_{1} \subset U$, because $\bar{U}_{1} \vdash Q$ by (i) and we have finished the proof of 1.2.

1.4. Corollary. Let $Q \in\{$ local compactness, local pseudocompactness, local countable compactness, local Lindelöf property, local Souslin property, local separability, local $\sigma$-compactness, local network weight $\leq \tau$, local spread $\leq \tau$, local extent $\leq \tau$ \}. Then $Q$ is $n$-additive in $X^{n}$ for any regular space $X$.

1.5. Lemma. Let open sets be representative for a pointwise property $Q$ which is hereditary with respect to closed sets. Suppose that $n>1$ and it is known 
that $Q$ is $(n-1)$-additive in $X^{n-1}$ for every $X$. Let $X^{n}=\bigcup\left\{X_{i}: i \in n\right\}$ and $X_{i} \vdash Q$ for all $i \in n$. Take any $x \in X$ and $U \in T(x, X)$. If there exists $a$ $j \in n$ with $\left(\{y\} \times U^{n-1}\right) \cap X_{j}=0$ for some $y \in U$, then $X \vdash Q(x, X)$.

Proof. The subspace $\{y\} \times U^{n-1}$ is homeomorphic to $U^{n-1}$ and is covered by the sets $X_{i} \cap\left(\{y\} \times U^{n-1}\right), i \in n \backslash\{j\}$. The set $\{y\} \times \dot{U}^{n-1}$ is representative for $Q$ in $\{y\} \times X^{n-1}$ so that $X_{i} \cap\left(\{y\} \times U^{n-1}\right) \vdash Q$ for all $i \in n \backslash\{j\}$. As $Q$ is $(n-1)$-additive in $U^{n-1}$, we have $U \vdash Q$. Hence $U \vdash Q(x, U)$ and $X \vdash Q(x, X)$ the set $U$ being representative for $Q$ in $X$. Our lemma is proved.

1.6. Proposition. Let $Q$ be a pointwise property such that

(i) if $Y \vdash Q(x, Y), Y \subset X$, and $x \in Z \subset \bar{Y}$ then $Z \vdash Q(x, Z)$;

(ii) open sets are representative for $Q$.

Then $Q$ is $n$-additive in $X^{n}$ for every topological space $X$ and $n \in \omega \backslash\{0\}$.

Proof. Take any $n>1$ and assume that we have proved $k$-additivity of $Q$ in $X^{k}$ for all $k \leq n-1$. Let $X^{n}=\bigcup\left\{X_{i}: i \in n\right\}$ and $X_{i} \vdash Q$ for every $i \in n$. Pick any $x \in X$ and consider the subspace $Y=\left\{\left(x^{n-1}, y\right): y \in X\right\}$. Clearly, $Y \simeq X$. There is a $j$ with $x^{n} \in X_{j}$. Lemma 1.5 shows that it suffices to prove $Q(x, X)$ in case $\left(V^{n-1} \times\{y\}\right) \cap X_{j} \neq 0$ for any $y \in X$ and $V \in T(x, X)$. But then $Y \subset \bar{X}_{j}$ so that $Q\left(x^{n}, X_{j}\right)$ and (i) imply $Q\left(x^{n}, Y\right)$. The last statement is equivalent to $Q(x, X)$ because there is a homeomorphism $h: Y \rightarrow X$ with $h\left(x^{n}\right)=x$. Then Proposition 1.6 is thus proved.

1.7. Corollary. The property "character $\leq \tau$ " is $n$-additive in $X^{n}$ for any $X \in$ $T_{3}$ and $n \geq 1$.

1.8. Proposition. Suppose that a pointwise $Q$ has the following properties:

(i) if $Q(x, X)$ and $x \in Y \subset X$ then $Q(x, Y)$;

(ii) if $H$ is representative for $Q$ in $X$ and $Y \subset X$ then $H \cap Y$ is representative for $Q$ in $Y$ and $H^{n}$ is representative for $Q$ in $X^{n}$;

(iii) if $y \in Y \subset X$ and $Q(y, Y)$ then there is a representative $H \subset X$ with $H \cap Y=\{y\}$

(iv) if $x^{n} \in H \subset X^{n}$ and $H$ is Q-representative in $X^{n}$ then there exists a $Q$-representative $G$ in $X$ such that $x^{n} \in G^{n} \subset H$;

(v) if $Q(x, X)$ does not hold, then $\{x\}$ is not representative in $X$.

Then $Q$ is $n$-additive in $X^{n}$ for every topological space $X$ and $n \geq 1$.

Proof. It is again induction along $n \in \omega$. Let $X^{n}=\bigcup\left\{X_{i}: i<n\right\}$ and $X_{i} \vdash Q$ for all $i<n$. Assume that $\neg Q(x, X)$ for some $x \in X$. Pick a $j \in n$ with $x^{n} \in X_{j}$. Using (iii) and (iv) find a representative $H \subset X, H \ni x$ such that $H^{n} \cap X_{j}=\left\{x^{n}\right\}$. It follows from (v) that $H \neq\{x\}$ so pick any $y \in H \backslash\{x\}$. Evidently, $\left(H^{n-1} \times\{y\}\right) \cap X_{j}=0$. Now (i) and (ii) make it possible to apply the induction hypothesis to the subspace $H$. Hence $H \vdash Q(x, H)$ and $X \vdash Q(x, X)$ which is a contradiction. Proposition 1.8 is proved.

1.9. Corollary. Pseudocharacter and discreteness are $n$-additive in $X^{n}$ for every $T_{1}$-space $X$ and $n \geq 1$.

Proof. Let $Q=$ " $\psi \leq \tau$ ". Then $G_{\tau}$-sets are representative for $Q$ and satisfy (i)(v). Proposition 1.8 applies for the discreteness as well because the discreteness is equivalent to the property $Q=$ " $\psi \leq 1$ ". 
1.10. Proposition. Suppose that we have pointwise properties $Q$ and $R$ such that

(i) $Q(x, X)$ and $x \in Y \subset X$ imply $Q(x, Y)$;

(ii) $R(x, X)$ and $x \in Y \subset X$ imply $R(x, Y)$;

(iii) any $U \in T^{*}(X)$ is representative for $Q$;

(iv) if $R(x, X)$ then $R\left(x^{n}, X^{n}\right)$;

(v) if $\neg Q(x, X)$ then there exists a $Y \subset X, Y \ni x$ with $\neg Q(x, Y)$ and $R(x, Y)$;

(vi) if $Q(x, Y)$ and $R(x, Y)$ then $\{x\} \in T(Y)$;

(vii) if $\neg Q(x, X)$ then $x \in \overline{X \backslash\{x\}}$.

Then $Q$ is $n$-additive in $X^{n}$ for any topological space $X$ and $n \geq 1$.

Proof. As usual it is an induction over $n \in \omega \backslash\{0\}$. Let $X^{n}=X_{0} \cup \cdots \cup X_{n-1}$ with $X_{i} \vdash Q$ for all $i \in n$. Assume that $\neg Q(x, X)$ for some $x \in X$. Use (v) to find a $Y \subset X, Y \ni x$ with $\neg Q(x, Y)$ and $R(x, Y)$. It follows from (i) that $Y_{i}=X_{i} \cap Y^{n} \vdash Q$ for every $i \in n$. If $x^{n} \in Y_{j}$ then (ii) and (iv) imply $R\left(x^{n}, Y_{j}\right)$. But $Y_{j} \vdash Q$ so that $Q\left(x^{n}, Y_{j}\right)$ takes place. By (vi) there is a $U \in T(x, Y)$ with $U^{n} \cap Y_{j}=\left\{x^{n}\right\}$. Pick any $y \in U \backslash\{x\}$ (which is possible by (vii)) and observe that $\left(U^{n-1} \times\{y\}\right) \cap Y_{j}=0$. Finally apply Lemma 1.5 to conclude that $Q(x, Y)$ holds which is a contradiction. Proposition 1.10 is proved.

1.11. Corollary. For any cardinal $\tau$ the $P_{\tau}$-property is $n$-additive in $X^{n}$ for $n \geq 1$ and $X \in T_{1}$.

Proof. Let $Q=$ " $P_{\tau}$-property" and $R=$ " $\psi \leq \tau$ ". If $x \in X$ is not a $P_{\tau}$-point in $X$ (i.e. $X \vdash \neg Q(x, X)$ ) then there is a $G_{\tau}$-set $H \ni x$ with $x \in \overline{X \backslash H}$. Now for the subspace $Y=\{x\} \cup(X \backslash H)$ we have $\psi(x, Y) \leq \tau$ (i.e. $Y \vdash R(x, Y)$ ) and $\neg Q(x, Y)$ so that (v) takes place. The proof of (i)-(iv) and (vi)-(vii) is trivial.

1.12. Theorem. Let a pointwise $Q$ have the following properties:

(i) open sets are representative for $Q$;

(ii) $X \vdash Q(x, X)$ iff $Q(x, A, X)$ for any $A \subset X$ with $x \in \bar{A}$;

(iii) $Q(x,\{x\}, X)$;

(iv) if $F$ is closed in $X$ and $x \in F$ then $Q(x, X)$ implies $Q(x, F)$;

(v) if $x \in Y \subset X, A \subset Y$, and $Q(x, A, Y)$ then $Q(x, A, X)$;

(vi) $A \subset B$ and $Q(x, A, X)$ imply $Q(x, B, X)$;

(vii) if $f: X \rightarrow X_{1}$ is a continuous map and $Q(x, A, X)$ then we have $Q\left(f(x), f(A), X_{1}\right)$.

Then $Q$ is $n$-additive in $X^{n}$ for every natural $n \geq 1$ and $X \in T_{3}$.

Proof. If $\neg(X \vdash Q)$ then there is an $x \in X$ with $\neg Q(x, X)$. Therefore $\neg Q(x, A, X)$ for some $A \subset X$ with $x \in \bar{A}$. Observe also that $x \not \supset A$ by (iii) and (iv). Let $X^{n}=X_{0} \cup \cdots \cup X_{n-1}$ and $X_{i} \vdash Q$ for all $i \in n$. Take a $j \in n$ with $x^{n} \in X_{j}$.

Claim. For every $y \in A$ we have

$$
\overline{X_{j} \cap p_{n}^{-1}(y)} \ni\left(x^{n-1}, y\right)
$$

where $p_{n}: X^{n} \rightarrow X$ is the natural projection onto the last factor. 
Proof of the claim. If $\overline{X_{j} \cap p_{n}^{-1}(y)} \not \supset\left(x^{n-1}, y\right)$ then by regularity of $X$ there is a $U \in T(x, X)$ such that $\left(\bar{U}^{n-1} \times\{y\}\right) \cap X_{j}=0$. It follows from (iv) that $\left(X_{i} \cap \bar{U}^{n-1}\right) \vdash Q$ for all $i \in n$. But the space $\bar{U}^{n-1} \times\{y\}$ is homeomorphic to $\bar{U}^{n-1}$ and is covered by $\leq n-1$ subspaces having $Q$. Hence $\bar{U} \vdash Q$ by induction hypothesis. Now (i) implies $Q(x, U)$ which in its turn implies $Q(x, X)$. This contradiction proves our claim.

Let $D=X_{j} \cap p_{n}^{-1}(A)$. It is clear that $x^{n} \in \bar{D}$. Thus $Q\left(x^{n}, D, X_{j}\right)$ takes place, so that $Q\left(x^{n}, D, X^{n}\right)$ is true by (v). Finally, use (vii) to conclude that $Q\left(x, p_{n}(D), X\right)$ is fulfilled. But $p_{n}(D)=A$ by the claim and we have a contradiction which proves Thecrem 1.12.

1.13. Corollary. If $Q \in\{$ tightness $\leq \tau$, sequentiality, $F U$-property, radiality, pseudoradiality $\}$ then $Q$ is n-additive in $X^{n}$ for any regular $X$ and $n \geq 1$.

Proof. We are going to formulate explicitly what $Q(x, A, X)$ is for every $Q$ from the above list.

(i) $Q=" t \leq \tau$." Then $Q(x, A, X)$ says $x \in \bar{A}^{\tau}$.

(ii) If $Q$ is "sequentiality" and $A \subset X$ let $A_{0}=A$. Having some sets $A_{\alpha}$ for all $\alpha<\beta$ consider two cases:

Case 1. $\beta=\lim \beta$. Put $A_{\beta}=\bigcup\left\{A_{\alpha}: \alpha<\beta\right\}$.

Case 2. $\beta=\beta_{0}+1$. Let $A_{\beta}=\left\{\lim a_{n}:\left\{a_{n}: n \in \omega\right\} \subset A_{\beta_{0}}\right.$ is a converging sequence $\}$. Finally, let $\bar{A}^{s}=\bigcup\left\{A_{\alpha}: \alpha<\omega_{1}\right\}$. The statement $Q(x, A, X)$ is to say that $x \in \bar{A}^{s}$.

(iii) If $Q$ is " $F U$-property" then $Q(x, A, X)$ states that $x \in A_{1}$, where $A_{1}$ is taken from (ii).

(iv) Let $Q=$ "radiality" and $A \subset X$. Then $\bar{A}^{r}$ is the set of limits of well-ordered nets lying in $A$. Define the statement $Q(x, A, X)$ to be equal to “ $x \in \bar{A}^{r}$ ".

(v) If $Q$ is "pseudoradiality" and $A \subset X$ take $\tau=(\exp (\exp (|X|)))^{+}$and for every $\alpha<\tau$ construct $A_{\alpha}$ as follows.

Let $A_{0}=A$ and if $\beta=\lim \beta$ then $A_{\beta}=\bigcup\left\{A_{\alpha}: \alpha<\beta\right\}$. If $\beta=\beta_{0}+1$ then $A_{\beta}=\bar{A}_{\beta_{0}}^{r}$. The sets $A_{\alpha}, \alpha<\tau$, being constructed put $\bar{A}^{p r}=\bigcup\left\{A_{\alpha}: \alpha<\tau\right\}$. Now $Q(x, A, X)=$ " $x \in \bar{A}^{p r}$ ".

The verification of conditions $1.12(\mathrm{i})-(\mathrm{vii})$ in any of $(\mathrm{i})-(\mathrm{v})$ cases is routine so that 1.13 is established.

The following property could be called the intermediate between pointwise and nonpointwise, because it is local but not pointwise.

1.14. Proposition. The k-property is n-additive in $X^{n}$ for any $X \in T_{3}$ and $n \geq 1$.

Proof. Assume that $X$ is a counterexample, $X^{n}=\bigcup\left\{X_{i}: i \in n\right\}, X_{i}$ is a $k$ space for each $i \in n$ and $n$ is the minimal number for which a counterexample exists. Let $A \subset X, A \neq \bar{A}$ be $k$-closed in $X$ i.e. $K \cap A$ is compact for any compact $K \subset X$. Then $p_{1}^{-1}(A)$ is $k$-closed in $X^{n}$. Pick any $a \in \bar{A} \backslash A$ and $j \in n$ with $a^{n} \in X_{j}$. The set $p_{1}^{-1}(A) \cap X_{j}$ is closed in $X_{j}$ so that there is a $U \in T(a, X)$ such that $\bar{U}^{n} \cap \overline{X_{j} \cap p_{1}^{-1}(A)}=0$. Take a $y \in U \cap A$. Then 
$\{y\} \times U^{n-1} \subset p_{1}^{-1}(A)$ and $\left(\{y\} \times U^{n-1}\right) \cap X_{j}=0$. This means $U^{n-1}$ is a union of $\leq n-1 \quad k$-spaces and is therefore a $k$-space itself. But this is a contradiction because $A \cap U$ is $k$-closed in $U$ but $\overline{A \cap U}^{U} \neq A \cap U$.

Proposition 1.14 is proved.

1.15. Theorem. The weight is $n$-additive in $X^{n}$ for every $X \in T_{3}$ and $n \geq 1$. Proof. It is again induction along $n \in \omega$. Let $n>1$ and $X^{n}=\bigcup\left\{X_{i}: i \in n\right\}$ where $w\left(X_{i}\right) \leq \tau$. We would like to reduce our case to an $X$ as simple as possible.

1.16. Lemma. If a counterexample exists then there is a counterexample $X$ such that $X=A \cup B$, where $A \cap B=0,|A| \leq \tau, w(B) \leq \tau$, and $\bar{A}=X$.

Proof of the lemma. Note that $\chi(X) \leq \tau$ by 1.7. If $X$ is some counterexample then $X=Y_{0} \cup \cdots \cup Y_{n-1}$ and $w\left(Y_{i}\right) \leq \tau$. Consider the subspaces $Z_{0}=$ $Y_{0}, \ldots, Z_{k}=Z_{k-1} \cup Y_{k}$. There must be an $i \in n$ such that $w\left(Z_{i}\right) \leq \tau$ but $w\left(Z_{i+1}\right)>\tau$. Hence $Z_{i+1}$ is also a counterexample and is a union of two subspaces of weight $\leq \tau$.

1.17. Sublemma. Let $Y=Y_{0} \cup \cdots \cup Y_{k}, w\left(Y_{i}\right) \leq \tau$, and $\bar{Y}_{i}=Y$ for all $i \leq k$. Then $w(Y) \leq \tau$.

Proof of sublemma. If $C_{i}$ is a base in $Y_{i},\left|C_{i}\right| \leq \tau$ then pick any $B_{i} \subset T(Y)$ such that $\left|B_{i}\right| \leq \tau$ and $C_{i}=B_{i} \mid Y_{i}$. Then $B=\bigcup\left\{B_{i}: i \leq k\right\}$ is a base in $Y$.

The sublemma being proved consider $Z_{i+1}=Z_{i} \cup Y_{i+1}$. It follows from $w\left(Z_{i+1}\right)>\tau$ that $w\left(\operatorname{cl}_{Z_{i+1}}\left(Z_{i}\right)\right)>\tau$ or $w\left(\mathrm{cl}_{Z_{i+1}}\left(Y_{i+1}\right)\right)>\tau$. If, for example $w\left(\bar{Z}_{i} \cap Z_{i+1}\right)>\tau$, then for $A_{1}=Z_{i}, B=\bar{Z}_{i} \cap Y_{i+1}$ the space $A_{1} \cup B$ is also a counterexample and $A_{1}$ is dense in $A_{1} \cup B$. Now take any $A \subset A_{1}$, $|A| \leq \tau$ such that $\bar{A} \supset A_{1}$ and let $X=A \cup B$. It follows from 1.17 that $X$ is a counterexample and Lemma 1.16 is proved.

1.18. Definition. Let $Y$ be a space and $P \subset Y$. A family $E$ is called an outer base of the set $P$ in $Y$ if for any $p \in P$ and $U \in T(p, Y)$ there is a $V \in E$ such that $p \in V \subset U$.

1.19. Lemma. Let $Y$ be a space, $Z \subset Y, T \subset Y, Z \cap T \neq 0$ and $T \subset \bar{Z}$, $w(Z) \leq \tau$. Then $Z \cap T$ has an outer base $E$ in $T$ with $|E| \leq \tau$.

Proof. Let $\gamma$ be a base in $Z,|\gamma| \leq \tau$. It follows from $\bar{Z} \supset Z \cup T$ that any family $\mu \subset T(Z \cup T)$ such that $\gamma=\mu\lceil Z$ will be an outer base of $Z$ in $Z \cup T$. Therefore it will be an outer base of $Z \cap T$ in $Z \cup T$. Of course the family $E=\mu \nmid T$ is an outer base of $Z \cap T$ in $T$ and Lemma 1.19 is proved.

1.20. Lemma. Let $Y=A \cup B$, where $|A| \leq \tau, \chi(Y) \leq \tau, \bar{A}=Y, w(B) \leq \tau$. Then $w(Y) \leq \tau$ iff $B$ has an outer base in $Y$ of power $\leq \tau$.

Proof. Only sufficiency needs to be proved. The set $A$ has an outer base $E_{A}$ with $\left|E_{A}\right| \leq \tau$ (because $\left.\chi(Y) \leq \tau\right)$. If $E_{B}$ is an outer base for $B$ in $Y$ and $\left|E_{B}\right| \leq \tau$, then $E=E_{A} \cup E_{B}$ is a base in $Y$ and $|E| \leq \tau$. Lemma 1.20 is thus proved.

1.21. Lemma. Let $Y=A \cup B, \chi(Y) \leq \tau,|A| \leq \tau, \bar{A}=Y, w(B) \leq \tau$, but $w(Y)>\tau$. Then there is a set $C \subset B$ such that $|C|=\tau^{+}$and $w(Z)>\tau$ for every $Z \supset A$ with $|Z \cap C|>\tau$.

Proof. We are going to construct $C$ by recursion along $\tau^{+}$. Let $\beta<\tau^{+}$and suppose we have $C_{\beta}=\left\{c_{\alpha}: \alpha \leq \beta\right\} \subset B$. We are assuming that a base $B_{y}$ of 
$T(y, Y)$ is chosen for any $y \in Y$ in such a way that $\left|B_{y}\right| \leq \tau$. The family $B_{\beta}=\bigcup\left\{B_{y}: y \in A \cup C_{\beta}\right\}$ is not a base in $Y$ so that there is a $c_{\beta+1} \in B$ such that $B_{\beta}$ is not a base at $c_{\beta+1}$. Put $C_{\beta+1}=C_{\beta} \cup\left\{c_{\beta+1}\right\}$. If $\alpha=\lim \alpha$, then $C_{\alpha}=\bigcup\left\{C_{\beta}: \beta<\alpha\right\}$.

Let us prove that the set $C=\left\{c_{\alpha}: \alpha<\tau^{+}\right\}$is as required. Take any $Z \supset$ $A,|Z \cap C|>\tau$. Suppose that $w(Z) \leq \tau$. Any base contains a subbase of minimal cardinality. Hence there is a $C^{\prime} \subset C,\left|C^{\prime}\right| \leq \tau$ such that the family $E=\bigcup\left\{B_{y}: y \in A \cup C^{\prime}\right\} \mid Z$ is a base in $Z$. There is an $\alpha<\tau^{+}$such that $c_{\alpha} \in Z$ and $\beta<\alpha$ as soon as $c_{\beta} \in C^{\prime}$. We have $\bar{Z}=Y$ so that the family $\mu=\bigcup\left\{B_{y}: y \in A \cup C^{\prime}\right\}$ is an outer base of $Z$. On the other hand $\mu$ is not a base at $c_{\alpha}$ by construction of $c_{\alpha}$. This contradiction proves Lemma 1.21.

Lemma 1.21 guarantees the existence of a counterexample $X$ such that

(*) $X=A \cup B,|A| \leq \tau, \bar{A}=X, w(B) \leq \tau,|B|=\tau^{+}$and $w(Z)>\tau$ for any $Z \supset A$ with $|Z \cap B|>\tau$.

Start at last the proof of Theorem 1.15. Let $X^{n}=X_{0} \cup \cdots \cup X_{n-1}$. Denote the set $\bar{X}_{0} \cap \cdots \cap \bar{X}_{n-1}$ by $P$. Suppose that $\left\{x^{n-1}\right\} \times X \not \subset P$ for all $x \in X$. Then any $x \in X$ has an $0 x \in T(x, X)$ such that $\left((0 x)^{n-1} \times\{p\}\right) \cap X_{i}=0$ for some $p \in X$ and $i \in n$. The induction hypothesis yields $w(0 x) \leq \tau$. Then $w(X) \leq \tau$ because $l(X) \leq n w(X) \leq \tau$ takes place.

Thus $\left\{x^{n-1}\right\} \times X \subset P$ for some $x \in X$. Moreover $\left\{x^{n-1}\right\} \times X \subset X_{0} \cup \cdots \cup$ $X_{n-1}$, so that the power of $Q_{i}=X_{i} \cap\left(\left\{x^{n-1}\right\} \times B\right)$ is greater than $\tau$ for some $i \in n$. We can apply Lemma 1.19 for $Y=X^{n}, Z=X_{i}, T=Q_{i} \cup\left(\left\{x^{n-1}\right\} \times A\right)$. We conclude that $Q_{i}$ has an outer base $E$ in $T$ with $|E| \leq \tau$. Lemma 1.20 implies $w(T) \leq \tau$ which contradicts $(*)$.

The proof of Theorem 1.15 is complete.

1.22. Theorem. If $c(Z)=\omega$ and $Z \in T_{3}$ then metrizability is $n$-additive in $Z^{n}$.

Proof. Note first that $\chi(Z)=\omega$ by 1.7. Next we will have to investigate a very weird class of spaces we are calling pyramids.

1.23. Definition. Given a first countable space $X$ we are going to define when the range $r(X) \in \omega$ makes sense. Let $r(X)=0$ iff $X=0$ and if $1 \leq|X| \leq \omega$ then $r(X)=1$.

Now $r(X)=n>1$ if $X=Y \cup Z, \bar{Z}=Z, Y \cap Z=0,|Y|=\omega, \bar{Y}=X$ and $Z=\bigoplus\left\{Z_{\alpha}: \alpha<\omega_{1}\right\}$ where $r\left(Z_{\alpha}\right)=n-1$ for all $\alpha<\omega_{1}$.

1.24. Remark. The range makes sense for a very narrow class of spaces. In what follows $r(X)=n$ will mean that the range of $X$ exists and is equal to $n$.

Let us describe in more detail a space $X$ with $r(X)=n>1$. We have by definition:

$X=V_{n} \cup Y_{n-1}, \quad V_{n} \cap Y_{n-1}=0, \bar{Y}_{n-1}=Y_{n-1},\left|V_{n}\right|=\omega, \bar{V}_{n}=X ;$ $Y_{n-1}=\bigoplus\left\{X_{n-1}^{\alpha_{1}}: \alpha_{1} \in \omega_{1}\right\}, X_{n-1}^{\alpha_{1}}=V_{n-1}^{\alpha_{1}} \cup Y_{n-2}^{\alpha_{1}}, V_{n-1}^{\alpha_{1}} \cap Y_{n-2}^{\alpha_{1}}=0, \overline{Y_{n-2}^{\alpha_{1}}}$ $=Y_{n-2}^{\alpha_{1}},\left|V_{n-1}^{\alpha_{1}}\right|=\omega, \overline{V_{n-1}^{\alpha_{1}}}=X_{n-1}^{\alpha_{1}}, Y_{n-2}^{\alpha_{1}}=\bigoplus\left\{X_{n-2}^{\alpha_{1} \alpha_{2}}: \alpha_{2} \in \omega_{1}\right\}, X_{n-2}^{\alpha_{1} \alpha_{2}}$ $=V_{n-2}^{\alpha_{1}-\alpha_{2}} \cup Y_{n-3}^{\alpha_{1} \alpha_{2}}, \ldots, X_{2}^{\alpha_{1} \cdots \alpha_{n-2}}=V_{2}^{\alpha_{1} \cdots \alpha_{n-2}} \cup Y_{1}^{\alpha_{1} \cdots \alpha_{n-2}}, V_{2}^{\alpha_{1} \cdots \alpha_{n-2}} \cap Y_{1}^{\alpha_{1} \cdots \alpha_{n-2}}$ $=0, \overline{Y_{1}^{\alpha_{1} \cdots \alpha_{n-2}}}=Y_{1}^{\alpha_{1} \cdots \alpha_{n-2}},\left|V_{2}^{\alpha_{1} \cdots \alpha_{n-2}}\right|=\omega, \overline{V_{2}^{\alpha_{1} \cdots \alpha_{n-2}}}=X_{2}^{\alpha_{1} \cdots \alpha_{n-2}}, Y_{1}^{\alpha_{1} \cdots \alpha_{n-2}}=$ $\bigoplus\left\{X_{1}^{\alpha_{1} \cdots \alpha_{n-2} \alpha_{n-1}}: \alpha_{n-1} \in \omega_{1}\right\}, r\left(X_{1}^{\alpha_{1} \cdots \alpha_{n-1}}\right)=1, V_{1}^{\alpha_{1} \cdots \alpha_{n-1}}=X_{1}^{\alpha_{1} \cdots \alpha_{n-1}}$.

It is clear now that it is natural to call $X$ a pyramid of range $n$ (or $n$ storey pyramid), and the set $V_{n}-$ the vertex of $X$. Let us define storeys 
of the pyramid $X$ in the following way: $E_{n}=\left\{V_{n}\right\}, E_{n-1}=\left\{V_{n-1}^{\alpha_{1}}: \alpha_{1} \in\right.$ $\left.\omega_{1}\right\}, \ldots, E_{n-k}=\left\{V_{n-k}^{\alpha_{1} \cdots \alpha_{k}}: \alpha_{1}, \ldots, \alpha_{k} \in \omega_{1}\right\}, \ldots, E_{1}=\left\{V_{1}^{\alpha_{1} \cdots \alpha_{n-1}}: \alpha_{1}, \ldots\right.$, $\left.\alpha_{n-1} \in \omega_{1}\right\}$.

We have therefore for whatever a space of range $n$, a unique decomposition of $X$ into storeys. This decomposition makes $X$ an $n$-storey pyramid. In the sequel symbols $V_{n}^{X}, E_{k}^{X}$ will be used if it is not clear from the context that the decomposition into the storeys is generated by the space $X$. An $m$-storey pyramid $Z$ is called a subpyramid of an $n$-storey pyramid $X$ if $Z$ is a subspace of $X$ and there exists a strictly increasing map $l=l(Z, X):\{1, \ldots, m\} \rightarrow$ $\{1, \ldots, n\}$ such that for every $P \in E_{k}^{Z}$ there is a $Q_{P} \in E_{l(k)}^{X}$ with $P \subset Q_{P}$ and the following takes place:

$$
\text { if } P_{1} \in E_{k_{1}}^{Z}, P_{2} \in E_{k_{2}}^{Z}, k_{1}<k_{2} \text {, then } P_{1} \subset \bar{P}_{2} \text { iff } Q_{P_{1}} \subset \overline{Q_{P_{2}}}
$$

(every bar denotes closure in relevant space). The simplest examples of subpyramids of $X$ are the pyramids $X_{n-k}^{\alpha_{1} \cdots \alpha_{k}}$ with vertices $V_{n-k}^{\alpha_{1} \cdots \alpha_{k}}$. Another example is $X$ with its $k$ th storey deleted $(k<n)$.

1.25. Lemma. Let $X$ be an $n$-storey pyramid, where $n>1$. If $M \subset X$ is metrizable then $M$ is a discrete sum of spaces of range 1 .

Proof. It is easy to show that every uncountable subset of $X$ has uncountable extent. Hence $\overline{M \cap V}_{n}^{M}$ is countable. The space $Y_{n-1}$ is an uncountable discrete sum of $(n-1)$-storey pyramids so that induction hypothesis can be used for $M \cap Y_{n-1}$. The rest is easy.

1.26. Lemma. Let $r(X)=n$ and let $M \subset X$ be metrizable. Then $X \backslash M$ has a subpyramid (in $X$ ) of range $n-1$.

Proof. Clearly, if $X_{1}$ is an $n$-storey subpyramid of $X$ then we may prove the lemma for $X_{1}$. That is why we will several times consider some $X_{1}$ instead of $X$ and we are going to denote these $X_{1}$ 's as $X$ again.

Let us consider the following subfamilies of $E_{1}: D_{11}=\left\{P \in E_{1}: P \subset M\right\}$, $D_{12}=E_{1} \backslash D_{11}$. We are going to show that at least one of the following conditions is fulfilled:

$\left(i_{1}\right)$ there exists an $n$-storey subpyramid $T \subset X$ such that $E_{1}^{T} \subset D_{11}$;

(iii $)$ there exists an $n$-storey subpyramid $T$ in $X$ such that $P \in E_{1}^{T}$ implies $Q_{P} \in D_{12}$ and $P \cap M=0$.

Let $t: \bigcup\left\{E_{i}: i \in n \backslash\{0\}\right\} \rightarrow \bigcup\left\{E_{i}: i \in(n+1) \backslash 1\right\}$ be a map defined as follows:

if $P \in E_{k}$, then $t(P) \in E_{k+1}, P \subset \overline{t(P)}$;

if $D \subset E_{k}$, then $u(D)=\left\{t(P): P \in D\right.$ and $\left.\left|t^{-1}(t(P)) \cap D\right|>\omega\right\}$.

It is immediate that $u(D) \cup u\left(E_{k} \backslash D\right)=E_{k+1}$ for every $D \subset E_{k}$. Now there exists an $n$-storey subpyramid $T \subset X$ with $E_{1}^{T} \subset D_{11}$ iff $\left|D_{11}^{1}\right|=\omega_{1}=\left|D_{11}^{2}\right|=$ $\left|D_{11}^{3}\right|=\cdots=\left|D_{11}^{n-1}\right|$, where $D_{1 p}^{1}=D_{1 p}, D_{1 p}^{i+1}=u\left(D_{1 p}^{i}\right), p=1,2, i=$ $1, \ldots, n-2$.

Suppose that $\left|D_{11}^{k}\right| \leq \omega$ for some $k \leq n-1$. Then $\left|D_{12}^{l}\right|=\omega_{1}$ for all $l=$ $1, \ldots, n-1$ because otherwise there would be an $m \leq n-1$ with $\left|D_{11}^{m}\right| \leq \omega$, $\left|D_{12}^{m}\right| \leq \omega$-we have a contradiction with $E_{m}=D_{11}^{m} \cup D_{12}^{m}$. Finally, to produce a subpyramid $T \subset X$ like in $\left(\mathrm{ii}_{1}\right)$ we have to put $E_{1}^{T}=\left\{P \backslash M: P \in D_{12}\right.$ and $P^{i} \in D_{12}^{i}$ for all $\left.i=1, \ldots, n-1\right\}$, where $P^{1}=P, P^{i+1}=t\left(P^{i}\right)$. If 
$1<k \leq n-1$, then $E_{k}^{T}=\left\{P \in D_{12}^{k}: P^{i} \in D_{12}^{k-1+i}\right.$ for $\left.i=1, \ldots, n-k\right\}$ and $E_{n}^{T}=\left\{V_{n}\right\}$.

We have proved that $\left(i_{1}\right)$ or $\left(i_{1}\right)$ takes place. They can also be fulfilled both at once. In that case we prefer $\left(\mathrm{ii}_{1}\right)$ and assume (without loss of generality) that the first storey of $X$ does not meet $M$. If only $\left(i_{1}\right)$ is true we will say that all elements of the first storey are subsets of $M$.

We are going to pass through all other storeys of our pyramid, considering smaller and smaller $n$-storey subpyramids (and saying all of them are equal to $X$ !). In the end we will have either an $n$-storey subpyramid outside of $M$ or an $n$-storey subpyramid $T$ with $\bigcup E_{k}^{T} \subset M$ for some $k \in\{1, \ldots, n\}$ and with $\left(T \backslash\left(\bigcup E_{k}^{T}\right)\right) \cap M=0$. This will finish the proof because then $T \backslash\left(\bigcup E_{k}^{T}\right)$ will be the required $(n-1)$-storey subpyramid outside $M$.

Suppose that first $k-1$ storeys are considered and $\left(\bigcup\left(\bigcup_{i=1}^{k-1} E_{i}\right)\right) \cap M=0$. Split $E_{k}$ into two parts: $D_{k 1}=\left\{P \in E_{k}: \overline{P \cap M}\right.$ contains a subpyramid of range $k\}$ and $D_{k 2}=E_{k} \backslash D_{k 1}$.

We will prove that at least one of the following conditions is fulfilled:

$\left(\mathrm{i}_{k}\right)$ there is a subpyramid $T \subset X$ with $r(T)=n$ and $\bigcup E_{k}^{T} \subset M$;

$\left(\mathrm{ii}_{k}\right)$ there is a subpyramid $T \subset X$ with $r(T)=n$ and $\left(\bigcup E_{k}^{T}\right) \cap M=0$.

This means we can either clear up the $k$ th storey from $M$ or take it that $M$ covers the whole $k$ th storey.

To start with observe that if $P \in E_{k}, A \subset P$ then either $\bar{A}$ or $\overline{P \backslash A}$ contains a $k$-storey subpyramid. This can be proved by easy induction along $k$. Let $D_{k p}^{1}=D_{k p}, D_{k p}^{i+1}=u\left(D_{k p}^{i}\right), i=1, \ldots, n-k-1, p=1,2$.

It is clear that there is an $n$-storey subpyramid $T \subset X$ such that $E_{k}^{T} \subset D_{k 1}$ iff $\left|D_{k 1}^{i}\right|=\omega_{1}, i=1, \ldots, n-k$. It follows from $E_{k+i-1}^{X}=D_{k 1}^{i} \cup D_{k 2}^{i}$ that either $E_{k}^{T} \subset D_{k 1}$ or $E_{k}^{T} \subset D_{k 2}$ for some $n$-storey subpyramid $T \subset X$. If $E_{k}^{T} \subset D_{k 1}$ then change every $P \in D_{k 1}$ inio $P \cap M$. Then $T^{\prime} \subset T$ thus obtained is also an $n$-storey subpyramid of $X$ (note for the sake of accuracy that $E_{j}^{T^{\prime}}=E_{j}^{T}$ for $j \neq k$ and $\left.E_{k}^{T^{\prime}}=\left\{P \cap M: P \in E_{k}^{T}\right\}\right)$.

Now passing from $X$ to $T^{\prime}$ we have the $k$ th storey covered by $M$. If $E_{k}^{T} \subset D_{k 2}$ then changing every $P \in D_{k 2}$ into $P \backslash M$ we can clear up the $k$ th storey of $X$.

Finally, if there is a $k \in\{1, \ldots, n-1\}$ such that the $k$ th storey is covered by $M$, the $(k+l)$ th storey is under consideration and $i<k+l, i \neq k$ implies $\left(\bigcup E_{i}\right) \cap M=0$, then we must prove that $(k+l)$ th storey can also be cleared up.

Indeed, if $P \in E_{k+l}$, then $(\overline{M \cap P}) \cap\left(\bigcup E_{k}\right)$ is countable because $M$ is metrizable and Lemma 1.25 implies $\left|\bar{A}^{M}\right|=\omega$ for any countable $A \subset M$.

Thus $\overline{P \backslash M}$ contains a subpyramid of range $k+l$ and the operation $P \rightarrow$ $P \backslash M$ clears up the $(k+l)$ th storey.

Lemma 1.26 is at last proved.

1.27. Corollary. No n-storey pyramid can be represented as a union of less than $n$ metric spaces.

It is time to set to the proof of Theorem 1.22.

Let $Z^{n}=Z_{0} \cup \cdots \cup Z_{n-1}, c(Z)=\omega$ and $Z_{i}$ is metrizable for all $i \in n$. If $s(Z)=\omega$ then $n w(Z)=\omega$ the space $Z$ beirig a finite union of separa- 
ble metrizable spaces. Therefore $w\left(Z_{i}\right)=n w\left(Z_{i}\right)=\omega$ and $w(Z)=\omega$ by Theorem 1.15.

Suppose $D \subset Z$ is discrete, $|D|=\omega_{1}$. If $Z$ is a counterexample then $Z \backslash(\bar{D} \backslash D)$ is also a counterexample because $c(Z \backslash(\bar{D} \backslash D))=\omega$. Consequently, we can assume that $\bar{D}=D$.

Evidently, $Z=Y_{0} \cup \cdots \cup Y_{n-1}$ for some metrizable $Y_{i}, i \in n$. Let $U_{i}=$ $\operatorname{Int}\left(\overline{Y_{i}}\right), W_{i}=Y_{i} \cap U_{i}$. Then $\bar{W}_{i}=\bar{U}_{i}$, and $c\left(W_{i}\right)=c\left(U_{i}\right)=\omega=d\left(W_{i}\right)$. As $\bigcup\left\{W_{i}: i \in n\right\}$ is dense in $Z$ we have $d(Z)=\omega$. Take $A \subset Z,|A|=\omega$, $\bar{A}=Z$. The space $A \cup D$ is still a counterexample so that again we are assuming $Z=A \cup D, A \cap D=0$.

Claim. $Z^{n}$ contains an $n$-storey pyramid.

Proof of the claim. Induction on $n$. Clearly, $Z^{1}=Z$ is a pyramid of range 2 . Let $X_{n-1} \subset Z^{n-1}, r\left(X_{n-1}\right)=n$. Then $X_{n}=A^{n} \cup\left(X_{n-1} \times D\right)$ is a pyramid of range $n+1$ and the claim is proved.

Now use Corollary 1.27 to conclude that the pyramid $X_{n}$ and hence $Z^{n} \supset X_{n}$ cannot be represented as a union of $n$ metric spaces which is a contradiction. Theorem 1.22 is proved at last.

1.28. Theorem. Metrizability is $n$-additive in $X^{n}$ if $X$ is compact.

Proof. We will need a lemma, generalizing the analogous one belonging to $M$. G. Tkačenko [2].

1.29. Lemma. Let $X$ be a compact space, $X=\bigcup\left\{Y_{l}: l \leq k\right\}$ where $Y_{l}$ is metrizable $(l \leq k)$. Then $F=\bigcap\left\{\bar{Y}_{l}: l \leq k\right\}$ is metrizable.

Proof of the lemma. Let us first establish that $F$ is $\omega$-monolithic. Let $A \subset F$, $|A|=\omega, A_{i}=\bar{A} \cap Y_{i}$. It is sufficient to prove that $w\left(A_{i}\right)=\omega$ for all $i \leq k$. Fix $j \leq k$. Then $A_{j}=\left(\bigcup\left\{\overline{A \cap Y}_{i}: i \leq k\right\}\right) \cap Y_{j}$. Thus it suffices to prove that $\overline{A \cap Y}_{i} \cap Y_{j}$ is second countable for all $i \leq k$. The tightness of $X$ is countable [3] so that there is a $B \subset Y_{j}$ with $|B| \leq \omega$ and $\bar{B} \supset A$. Therefore $\overline{A \cap Y}_{i} \cap Y_{j} \subset \bar{B} \cap Y_{j}=\operatorname{cl}_{Y_{j}}(B)$. As $B$ is countable and $Y_{j}$ metrizable we have $w\left(\operatorname{cl}_{Y_{j}}(B)\right) \leq \omega$.

Hence the same is true for $\overline{A \cap Y}_{i} \cap Y_{j}$ and we have established $\omega$-monolithity of $F$.

The second step in the lemma proof is to show that $d(F)=\omega$.

Fix $E_{i} \subset T(X)$ such that $E_{i} \uparrow Y_{i}$ is a $\sigma$-discrete base in $Y_{i}$. Observe that $E_{i} \uparrow \bar{Y}_{i}$ is an outer base of $Y_{i}$ in $\bar{Y}_{i}$.

Let $A_{0}=\left\{x_{0}\right\}$, where $x_{0} \in F$. There exist countable sets $A_{0}^{i} \subset Y_{i}$ such that $A_{0} \subset \overline{A_{0}^{i}}$ for all $i \leq k$. Let $C_{0}(i)=\bigcup\left\{E_{i}(x): x \in A_{0}^{i}\right\}$ and $C_{0}=$ $C_{0}(0) \cup \cdots \cup C_{0}(k)$.

Suppose that $n \in \omega$ and we have countable sets $A_{n} \subset F, A_{n}^{i} \subset Y_{i}$ and countable $C_{n} \subset T(X), \bar{A}_{n} \subset \cup C_{n}$. For every finite $d \subset C_{n}$ with $\bar{A}_{n} \subset \bigcup d$ pick a point $x_{d} \in F \backslash \bigcup d$ if the last set is not empty. Put $A_{n+1}=A_{n} \cup\left\{x_{d}: d \subset\right.$ $C_{n}, \bar{A}_{n} \subset \cup d$ and $\left.F \backslash \bigcup d \neq 0\right\}$. Then $A_{n+1} \subset F$ and $\left|A_{n+1}\right| \leq \omega$. There are countable $A_{n+1}^{i} \subset Y_{i}$ such that $A_{n+1} \subset \overline{A_{n+1}^{i}}$ for all $i \leq k$. Let $C_{n+1}(i)=$ $\bigcup\left\{E_{i}(x): x \in A_{n+1}^{i}\right\}$ and $C_{n+1}=C_{n+1}(0) \cup \cdots \cup C_{n+1}(k)$. Our construction is complete. Let $A=\bigcup\left\{A_{n}: n \in \omega\right\}$ and $C=\bigcup\left\{C_{n}: n \in \omega\right\}, A(i)=\bar{A} \cap Y_{i}$, $i \leq k$. 
Claim. $C \supset C(0) \cup \cdots \cup C(k)$, where $C(i)=\bigcup\left\{E_{i}(x): x \in A(i)\right\}$.

Proof of the claim. If $x \in A(i), V \in E_{i}(x)$ then $V \cap A_{m}^{i} \neq 0$ for some $m \in \omega$ because $x \in \bar{A} \subset \overline{U\left\{A_{n}: n \in \omega\right\}}$. But then $V \in C_{m}(i) \subset C$. Hence $C(0) \cup \cdots \cup$ $C(k) \subset C$ and our claim is proved.

It follows from the claim that $C(x) \mid \bar{Y}_{i}$ is a base at $x$ in $\bar{Y}_{i}$ for any $x \in A(i), i \leq k$.

The set $A$ is dense in $F$. To prove that, suppose that this is not so. Then there is a point $z \in F \backslash \bar{A}$. Every $x \in \bar{A}$ has a neighbourhood $V(x) \in C(x)$ such that $z \notin V(x)$. The cover $\{V(x): x \in \bar{A}\}$ of the compact set $\bar{A}$ has a finite subcover $d$. There is an $m \in \omega$ with $d \subset C_{m}$. Then $\bar{A}_{m} \subset \bigcup d$ and $z \in F \backslash \bigcup d$. Thus there is an $x_{d} \in F \backslash \bigcup d$. But $x_{d} \in A_{m+1} \subset A$ and $x_{d} \in F \backslash \bigcup d \subset F \backslash \bar{A}$. This contradiction proves that $\bar{A}=F$. The separability of the set $F$ together with its monolithity implies $w(F) \leq \omega$ and Lemma 1.29 is proved.

Let $X^{n}=\bigcup\left\{X_{i}: i<n\right\}$ with metrizable $X_{i}, i<n$. Lemma 1.29 gives $w(F) \leq \omega$ for $F=\bigcap\left\{\bar{X}_{i}: i<n\right\}$. If $p_{0}(F)=X$ then, evidently, $X$ is metrizable. If $x \in p_{0}(F)$, then $p_{0}^{-1}(x) \cap F=0$ and $p_{0}^{-1}(x) \simeq X^{n-1}$. Now $X^{n-1}$ is represented as a union of $n$ metrizable spaces with empty intersection of their closures. Let $\Delta=\left\{y^{n-1}: y \in X\right\}$ be the diagonal of $X^{n-1}$. For every $y \in X$ there is a $U_{y} \in T(y, X)$ such that $\bar{U}_{y}^{n-1}$ meets less or equal than $n-1$ metrizable spaces. The induction hypothesis yields $w\left(U_{y}\right)=\omega$. Hence $X$ is locally metrizable and thus metrizable.

Then Theorem 1.28 is proved.

1.30. Theorem. The ANR-property is closely n-additive in $X^{n}$ for a compact space $X$.

Proof. In the class of compact spaces the ANR-property coincides with local ANR-property. Suppose that $X^{n}=\bigcup\left\{X_{i}: i \in n\right\}, X_{i}$ is closed in $X^{n}$ and is an ANR. We are gong to prove that $X$ is a local ANR.

Our induction hypothesis will be a bit stronger:

$\left(\mathrm{I}_{k}\right)$ if $X^{k}$ is embedded in $X^{p}$ as a $k$-face for some $p \geq k$ and $X^{k} \subset$ $\bigcup\left\{X_{i}: i<k\right\}, X_{i}$ is closed in $X^{p}$ and is an ANR then $X$ is locally ANR.

Recall that $Z \subset X^{p}$ is a $k$-face of $X^{p}$ if there are $x_{1}, \ldots, x_{p-k} X$ and $i_{1}, \ldots, i_{p-k} \in p$ such that $Z=\left\{f \in X^{p}: f\left(i_{l}\right)=x_{l}, l=1, \ldots, p-k\right\}$.

The statement $\left(\mathrm{I}_{1}\right)$ is true, because if $Z$ is a 1 -face of some $X^{p}$ and $Z \subset X_{0}$, then $Z$ is a retract of $X^{p}$ and thus a retract of $X_{0}$. A retract of an ANR is itself an ANR and we established $\left(I_{1}\right)$.

If we are questioning $\left(\mathrm{I}_{n}\right)$, take any $x \in X$. The space $X^{n}$ is an $n$-face of some $X^{q}$ and $X^{n} \subset \bigcup\left\{X_{i}: i<n\right\}$. Let $X^{n}=\left\{f \in X^{q}: f\left(i_{l}\right)=x_{l}\right.$, $l=1, \ldots, q-n, x_{l} \in X$ for all $\left.l\right\}$. Pick some $j \notin\left\{i_{1}, \ldots, i_{q-n}\right\}$ and let $F=p_{j}^{-1}(x) \cap X^{n}$, where $p_{j}: X^{q} \rightarrow X$ is the $j$ th projection. Then $F \simeq X^{n-1}$ and $F$ is an $(n-1)$-face of $X^{q}$. If $F \subset X_{0} \cup \cdots \cup X_{n-2}$ then by $\left(I_{n-1}\right)$ the space $X$ is an ANR. If not let $f \in F \backslash\left(X_{0} \cup \cdots \cup X_{n-2}\right)$. Pick a standard $U \in T\left(f, X^{q}\right)$ with $U \cap\left(X_{0} \cup \cdots \cup X_{n-2}\right)=0$. The set $U \cap X_{n-1}$ is an ANR being open in $X_{n-1}$. There is a retraction $r: U \cap X_{n-1} \rightarrow U_{1}$ where $U_{1} \ni f$ is a standard open set in $X^{n}$. Clearly, $U_{1}$ can be retracted onto a set homeomorphic to a neighbourhood of $x$. Thus $X$ is locally ANR at any $x \in X$ and $I_{n}$ is proved together with Theorem 1.30. 
1.31. Theorem. The dimension function ind is closely $n$-additive in $X^{n}$ for any regular $X$.

Proof. It runs like one in 1.30 but is simpler. If $X^{n}=\bigcup\left\{X_{i}: i \in n\right\}, X_{i}$ is closed in $X^{n}$ and $\operatorname{ind}\left(X_{i}\right) \leq k$ for all $i \in n$ then pick any $x \in X$. If $p_{0}^{-1}(x) \subset \bigcup\left\{X_{i}: i \in(n-1)\right\}$, then the induction hypothesis works (observe that we could not act in the same way proving 1.30 because the ANR-property is not closed hereditary). If not then $U \ni f \in p_{0}^{-1}(x)$ for some standard $U$ with $U \cap\left(\bigcup\left\{X_{i}: i<n-1\right\}\right)=0$. But $U \subset X_{n-1}$ and contains a copy of some $V \in T(x, X)$. Therefore ind $(V) \leq k$ and 1.31 is proved.

1.32. Examples. The space $L\left(\omega_{1}\right)$ shows that all properties we mentioned (except "ind $\leq k$ ") are not 2-additive. It is easy to prove that $\left(L\left(\omega_{1}\right)\right)^{n}$ is a union of $n+1$ discrete spaces. Therefore they are not $(n+1)$-additive in $\left(L\left(\omega_{1}\right)\right)^{n}$. It is well known that "ind $\leq 1$ " and ANR-property are not closely 2-additive [4]. There exists a metrizable compact space $X$ such that $\operatorname{ind}(X)=$ 2 , but $\operatorname{ind}\left(X^{2}\right)=3$ [5]. The last equality implies $X^{2}$ is a union of two 1dimensional spaces. But $\operatorname{ind}(X)>1$ so that ind is not 2-additive in $X^{2}$ even for metrizable compact $X$.

1.33. Questions. Let $Q \in$ \{normality, collectionwise normality, paracompactness, $i$-weight, $\pi$-character $\}$. Is $Q n$-additive in any $X^{n}$ ? Is Ind closely $n$-additive in $X^{n}$ (for, say compact $X$ ) ? Does there exist a property which is 17 -additive for $X^{17}$ but is not 18-additive for $X^{18}$ ?

\section{Countable adDitivity IN $X^{\omega}$}

Most of the properties are countably additive in $X^{\omega}$ for any Tychonoff $X$. More exactly, the following holds.

2.1. Proposition. Let $Q$ be a topological property invariant under open mappings. If $X \in T_{3.5}, X^{\omega}=\bigcup\left\{X_{i}: i \in \omega\right\}$ and $X_{i} \vdash Q$ for all $i \in \omega$, then $X \vdash Q$ (and $X^{\omega} \vdash Q$ ).

Proof. This was in fact proved by M. G. Tkačenko who had established the following

2.2. Lemma (M. G. Tkačenko [1]). If $X \in T_{3.5}$ and $X^{\omega}=\bigcup\left\{X_{i}: i \in \omega\right\}$ then there is a $j \in \omega$ such that $X_{j}$ can be mapped openly onto $X$.

It also follows from the Lemma 2.2 that some $X_{j}$ maps openly onto $X^{\omega}$ because $X^{\omega}=\left(X^{\omega}\right)^{\omega}$.

In [1] Tkačenko proved that metrizability is $\omega$-additive in $X^{\omega}$ if $\mathrm{cl}^{*}(X) \leq$ $\omega$, i.e. if any discrete $E \subset T^{*}\left(X^{n}\right)$ is countable for any $n \geq 1$.

2.3. Theorem. Metrizability is finitely additive in $X^{\omega}$ for any Tychonoff $X$.

Proof. Suppose that for all $k<n$ it is proved that if $X^{\omega}=\bigcup\left\{X_{i}: i<k\right\}$ and $\prod\left\{X_{i}: i<k\right\}$ is metrizable then so is $X$. If $X^{\omega}=\bigcup\left\{X_{i}: i<n\right\}$ then there - will be no loss of generality if we assume that every $X_{i}$ is strongly dense in $X^{\omega}$, i.e. for any finite $A \subset \omega$ we have $p_{A}\left(X_{i}\right)=X^{A}$.

Indeed, if some $X_{i}$ is not strongly dense in $X^{\omega}$ then take any $z \in X^{A} \backslash p_{A}\left(X_{i}\right)$ and apply the induction hypothesis to $p_{A}^{-1}(z)$ which is homeomorphic to $X^{\omega}$.

Let $B_{i}$ be a $\sigma$-discrete base in $X_{i}$ and let $E_{i} \subset T\left(X^{\omega}\right)$ satisfy $E_{i} \backslash X_{i}=B_{i}$. Then $E_{i}$ is an outer base for $X_{i}$ because $X_{i}$ is dense in $X^{\omega}$. We are going to 
produce a $\sigma$-discrete base for $E_{i}$, i.e. a family $D_{i} \subset T\left(X^{\omega}\right)$ such that $D_{i}$ is $\sigma$-discrete and for every $U \in E_{i}$ there is a $D_{U} \subset D_{i}$ with $\bigcup D_{U}=U$. If we succeed then $\bigcup_{i<n} D_{i}$ will be a $\sigma$-discrete tase in $X^{\omega}$.

We will apply the method M. G. Tkačenko used in [1]. If $U \in T\left(X^{\omega}\right)$ then let $U_{k}=p_{k}^{-1}\left(V_{k}\right)$, where $V_{k}=\bigcup\left\{W: W \in T\left(X^{k}\right)\right.$ and $\left.p_{k}^{-1}(W) \subset U\right\}$. Observe that $k$ is meant to be the set $\{0,1, \ldots, k-1\}$ and $p_{k}: X \rightarrow X^{k}$ is the relevant projection.

It is easy to check that $U=\bigcup\left\{U_{k}: k \in \omega\right\}$ for any $U \in T\left(X^{\omega}\right)$.

Represent $E_{i}$ as $\bigcup\left\{E_{i}^{s}: s \in \omega\right\}$, where $E_{i}^{s} \uparrow X_{i}$ is discrete in $X_{i}$. Consider the family $E_{i}^{s k}=\left\{U_{k}: U \in E_{i}^{s}\right\}$ for all $k \in \omega$. Fix a $z \in X^{k}$. There is a $y \in X_{i}$ with $p_{k}(y)=z$. Choose a $W \in T\left(X_{i}\right), W \ni y$ such that $W$ meets at most one element of $E_{i}^{s} \uparrow X_{i}$. Let $q_{k}=p_{k} \uparrow X_{i}$. The map $q_{k}$ is open [1], and the family $q_{k}^{-1}\left(p_{k}\left(E_{i}^{s k}\right)\right)$ refines the family $E_{i}^{s} \uparrow X_{i}$ so that $q_{k}(W)$ meets at most one element of $p_{k}\left(E_{i}^{s k}\right)$. We proved that $p_{k}\left(E_{i}^{s k}\right)$ is discrete in $X^{k}$. Therefore $E_{i}^{s k}$ is discrete too and letting $D_{i}=\bigcup\left\{E_{i}^{s k}: s, k \in \omega\right\}$ we cbtain a $\sigma$-discrete family which is a base of $E_{i}$.

Our proof is complete because $\bigcup\left\{D_{i}: i<n\right\}$ is a $\sigma$-discrete base in $X^{\omega}$ and $X^{\omega}$ is metrizable.

2.4. Theorem. Let $X$ be a $T_{1}$-space and $X^{\omega}=\bigcup\left\{X_{n}: n \in \omega\right\}$, where $\psi\left(X_{n}\right) \leq$ $\tau$ for all $n$. Then $\psi(X) \leq \tau$.

Proof. Note first that no technique, developed by M. G. Tkačenko in [1] can be applied here.

Suppose that $X$ is a counterexample. Then $X=\bigcup\left\{X_{n}^{\prime}: n<\omega\right\}$ where $\psi\left(X_{n}^{\prime}\right) \leq \tau$. There exist an $x \in X$ and an $n \in \omega$ with $\psi\left(x,\{x\} \cup X_{n}^{\prime}\right)>\tau$, i.e. the space $X^{\prime}=\{x\} \cup X_{n}^{\prime}$ is also a counterexample.

Consider the space $X^{\prime \prime}$ defined as follows: $X^{\prime \prime}=X^{\prime}$ and $T\left(X^{\prime \prime}\right)=\{\{y\}$ : $\left.y \in X_{n}^{\prime}\right\} \cup\left\{\bigcap_{\alpha<\tau} \cup \alpha: U_{\alpha} \in T(x, X)\right.$ for all $\left.\alpha<\tau\right\}$. This space is also a counterexample, because its topology is finer than that of $X^{\prime}$.

We proved that there will be no loss of generality if we assume $X=\{a\} \cup Y$, where $Y \subset T(X), a \in \bar{Y} \backslash Y, \psi(a, X)>\tau$, and if $U_{\alpha} \in T(a, X)$ for $\alpha<\tau$ then $\bigcap\left\{U_{\alpha}: \alpha<\tau\right\} \in T(a, X)$. For $A \subset \omega$ and $f \in X^{A}$ let $P(f)=\{n \in$ $A: f(n)=a\}, D=\left\{f \in X^{\omega}:|P(f)| \geq \omega\right\}$. The set $X^{\omega} \backslash D$ is a countable union of discrete spaces so that we can keep under consideration the set $D=$ $\bigcup\left\{X_{n}: n \in \omega\right\}$ only.

For any $n \in \omega$ construct $A_{n}, g_{n}, Z_{n}, k_{n}, U_{n}, y_{n}$, and $f_{n}$ with the following properties:

(1) $A_{n} \subset \omega, g_{n} \in X^{A_{n}}, k_{n} \in \omega, U_{n} \in T(a, X), y_{n} \in U_{n} \backslash\{a\}, f_{n} \in X^{\omega}$, $Z_{n} \subset D$

(2) $A_{n} \subset A_{n+1}, U_{n} \supset U_{n+1}, Z_{n} \supset Z_{n+1}, g_{n} \subset g_{n+1}, k_{n}<k_{n+1}$ for $n \in \omega$;

(3) $\left|P\left(g_{n}\right)\right|=n, f_{n} \mid A_{n}=g_{n}$;

(4) $Z_{n+1} \cap X_{k_{n}}=0, Z_{n} \cap X_{k_{n}}=\left\{f_{n}\right\}$.

Let $A_{0}^{\prime}=\{0,1\}, g_{0}^{\prime}(0)=a, g_{0}^{\prime}(1)=y_{0} \in Y$. Let $Z_{0}=\left(\left\{g_{0}^{\prime}\right\} \times X^{\omega \backslash 2}\right) \cap D$, $k_{0}=\min \left\{k: X_{k} \cap Z_{0} \neq 0\right\}$. Choose an $f_{0} \in X_{k_{0}} \cap Z_{0}$. It follows from $\psi\left(X_{k_{0}}\right) \leq$ $\tau$ that there exists a $U_{0} \in T(a, X)$ such that

$$
\left(U_{0}^{P\left(f_{0}\right)} \times\left\{f_{0}^{\prime}\right\}\right) \cap X_{k_{0}}=\left\{f_{0}\right\}, \quad \text { where } f_{0}^{\prime}=f_{0} \uparrow\left(\omega \backslash P\left(f_{0}\right)\right) .
$$

Then $A_{0}=A_{0}^{\prime} \cup\left(\omega \backslash P\left(f_{0}\right)\right), g_{0}=f_{0} \uparrow A_{0}$. 
Suppose we have $A_{m}, g_{m}, Z_{m}, k_{m}, U_{m}, y_{m}, f_{m}$ satisfying (1)-(4) for all $m \leq n$. It follows from (3) that the set $P\left(f_{n}\right) \backslash A_{n}$ is infinite. Take any different $l_{0}, l_{1} \in P\left(f_{n}\right) \backslash A_{n}$ and put $A_{n+1}^{\prime}=A_{n} \cup\left\{l_{0}, l_{1}\right\}$. Define $g_{n+1}^{\prime}$ in the following way: $g_{n+1}^{\prime}(k)=f_{n}(k), k \in A_{n}$ and $g_{n+1}\left(l_{0}\right)=a, g_{n+1}\left(l_{1}\right) y_{n+1} \in$ $U_{n} \backslash\{a\}$. Clearly, $g_{n} \subset g_{n+1}^{\prime}$. Let $Z_{n+1}=\left(\left\{g_{n+1}^{\prime}\right\} \times U_{n}^{\omega \backslash A_{n+1}^{\prime}}\right) \cap Z_{n}$ and $k_{n+1}=\min \left\{k: X_{k} \cap Z_{n+1} \neq 0\right\}$. Pick some $f_{n+1} \in X_{k_{n+1}} \cap Z_{n+1}$. It follows from $\psi\left(X_{k_{n+1}}\right) \leq \tau$ that there is a $U_{n+1} \in T\left(a, U_{n}\right)$ with

$$
\left(U_{n+1}^{P\left(f_{n+1}\right)} \times\left\{f_{n+1}^{\prime}\right\}\right) \cap X_{k_{n+1}}=\left\{f_{n+1}\right\},
$$

where $f_{n+1}^{\prime}=f_{n+1} \backslash\left(\omega \backslash P\left(f_{n+1}\right)\right)$. Now letting $A_{n+1}=A_{n+1}^{\prime} \cup\left(\omega \backslash P\left(f_{n+1}\right) g_{n+1}=\right.$ $f_{n+1}\left\lceil A_{n+1}\right.$ we have the inductive construction finished.

It is immediate that (1)-(3) and second part of (4) are fulfilled. But $Z_{n+1} \cap$ $X_{k_{n}}=0$ because $X_{k_{n}} \cap Z_{n}=\left\{f_{n}\right\}$ and $Z_{n+1} \subset Z_{n}, Z_{n+1} \not \supset f_{n}$. It is evident that $k_{n}=\min \left\{k: X_{k} \cap Z_{n} \neq 0\right\}$ for all $n \in \omega$. Thus

$$
\left(\bigcap\left\{Z_{n}: n \in \omega\right\}\right) \cap\left(\bigcup\left\{X_{n}: n \in \omega\right\}\right)=0
$$

i.e. $\bigcap\left\{Z_{n}: n \in \omega\right\}=0$. Let $g(k)=\left(\bigcup_{n \in \omega} g_{n}\right)(k)$ if $k \in \bigcup\left\{A_{n}: n \in \omega\right\}$ and $g(k)=a$ otherwise. Evidently, the point $g$ belongs to $\bigcap\left\{Z_{n}: n \in \omega\right\}$ and this contradiction proves our theorem.

\section{Finite AND COUNTABLE ADDITIVITY IN TOPOLOGICAL GROUPS}

A. V. Arhangel'skii proved [6] that if $G$ is a topological group, $G=X_{1} \cup$ $\cdots \cup X_{n}$ and $w\left(X_{i}\right) \leq \tau, i=1, \ldots, n$, then $w(G) \leq \tau$. This result seems to have inspired no one to prove that many other properties are finitely additive in groups.

3.1. Theorem. Let $M=\{$ metrizability, character, $\pi$-character, pseudocharacter, $P_{\tau}$-property, $i$-weight, pseudoweight, diagonal number local compactness, local pseudocompactness, local countable compactness $\}$. Suppose that $Q \in M$, $G$ is a topological group, $G=X_{0} \cup \cdots \cup X_{n-1}$ where $X_{i} \vdash Q$ for all $i \in n$. Then $G \vdash Q$ i.e. any $Q \in M$ is finitely additive in topological groups.

Proof. If $G=X_{0} \cup \cdots \cup X_{n-1}$ and $\pi \chi\left(X_{i}\right) \leq \tau$ for $i \in n$ then there is an $m \in n$ with $\bar{X}_{m} \supset U \in T^{*}(G)$. Hence $\pi \chi(g, U) \leq \pi \chi\left(X_{m}\right) \leq \tau$ for any $g \in X_{m} \cap U$. Therefore $\pi \chi(G) \leq \tau$. This proves finite additivity of the first three properties because $\pi \chi(G)=\chi(G)$ for any $G$ and $G$ is metrizable iff $\chi(G)=\omega$.

Suppose that $\psi\left(X_{i}\right) \leq \tau, i \in n$, and $e \in X_{j}$. There exists a $G_{\tau}$-subgroup $H \subset G$ with $H \cap X_{j}=\{e\}$. If $H \subset X_{j}$ then $H=\{e\}$ and the proof is over. If not, then pick any $g \in H \backslash X_{j}$ and a $G_{\tau}$-subgroup $H_{1} \subset H$ such that $g H_{1} \cap X_{j}=0$. Now $g H_{1} \simeq H_{1}$ and the induction hypothesis may be applied to $H_{1}$. This yields $\psi\left(H_{1}\right) \leq \tau$ and $\psi(G) \leq \tau$. We have actually proved our theorem for pseudocharacter and diagonal number because $\Delta(G)=\psi(G)$ for any $G[6]$.

If $p w\left(X_{i}\right) \leq \tau$ then $\left|X_{i}\right| \leq 2^{\tau}$ and thus $|G| \leq 2^{\tau}$. We have $\psi(G) \leq \tau$ by finite additivity of pseudocharacter. It is standard that there exists a one-toone continuous map $f: G \rightarrow \prod\left\{M_{\alpha}: \alpha<\tau\right\}$ where $M_{\alpha}$ is metrizable for all $\alpha<\tau$ and $\left|M_{\alpha}\right|<2^{\tau}$. There is a one-to-one continuous map $f_{\alpha}: M_{\alpha} \rightarrow N_{\alpha}$ where $w\left(N_{\alpha}\right) \leq \tau$ for all $\alpha<\tau$ [7]. Therefore $i w(G) \leq i w\left(\prod\left\{M_{\alpha}: \alpha<\tau\right\}\right) \leq$ 
$w\left(\Pi\left\{N_{\alpha}: \alpha<\tau\right\}\right) \leq \tau$ and we proved finite additivity of pseudoweight and $i$ weight at once. Clearly, we have also established the following fact which seems to be new.

\subsection{Corollary. If $G$ is a topological group, then $i w(G)=p w(G)$.}

Let us go on with the proof of 3.1. If $X_{i}$ is a $P_{\tau}$-space for all $i \in n$ then some $X_{i}$ is dense in a nonempty open set $U \subset G$. There will be no loss of generality to assume that $e \in X_{i}, U \in T(e, G)$. It suffices to prove that any $G_{\tau}$-subgroup $H \subset U$ is open which is equivalent to $\operatorname{Int}(H) \neq 0$.

There exists a closed $G_{\tau}$-subgroup $H_{1} \subset H$. We have $\operatorname{Int}_{X_{i}}\left(H_{1} \cap X_{i}\right) \neq Q$.

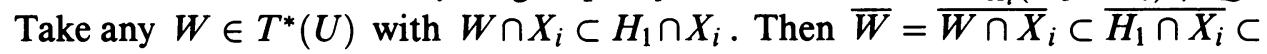
$\bar{H}_{1}=H_{1}$ so that $\operatorname{Int}\left(H_{1}\right) \neq 0$ and we are done with the $P_{\tau}$-property.

If $X_{i} \vdash Q_{\mathrm{loc}}$ (see Definition 1.1) and $Q_{\mathrm{loc}}$ is one of the three remaining properties then choose an open $U \subset G, U \neq 0$ such that $X_{i} \cap U \neq 0$ implies $\overline{X_{i} \cap U} \supset U$ for any $i$. There will be no loss of generality to assume that every $X_{i}$ is dense in $U$. Take $U_{0} \in T^{*}(U), \bar{U}_{0} \subset U$ with $\left(\bar{U}_{0} \cap X_{0}\right) \vdash Q$. If we have $U_{0} \supset U_{1} \supset \cdots \supset U_{k}$ pick some $U_{k+1} \in T^{*}\left(U_{k}\right), \bar{U}_{k+1} \subset U_{k}$ such that $\left(\bar{U}_{k+1} \cap X_{k+1}\right) \vdash Q$. After $n$ steps we obtain the set $U_{n} \in T^{*}(G)$ which is a union of $n$ subspaces having $Q$. Consequently, $\bar{U}_{n} \vdash Q$ so that $G \vdash Q_{\mathrm{loc}}$ and the Theorem 3.1 is proved.

3.3. Examples. Let $N=\{$ tightness, $F U$-property, sequentiality, $k$-property, normality\}. If $Q \in N$ then $Q$ is not 2-additive in topological Hausdorff groups.

Proof. Consider the group $2^{\omega_{1}}$ and its subgroup $H=\left\{f \in 2^{\omega_{1}}: \mid\{\alpha: f(\alpha)=\right.$ $1\} \mid \leq \omega\}$. Pick $f_{1} \in 2^{\omega_{1}}$ with $f_{1}(\alpha)=1$ for all $\alpha$. Then $G=H \cup\left(H+f_{1}\right)$ is a subgroup of $2^{\omega_{1}}$ covering almost all properties we must prove to be non 2-additive. Indeed $H$ and $H+f_{1}$ are $F U$-subspaces of $G$ but $t(G)=\omega_{1}$. It is well known that $H$ and $H+f_{1}$ are collectionwise normal. However, $G$ is not normal. To prove this take any $h \in 2^{\omega_{1}} \backslash G$. Construct $h_{\alpha}^{0} \in H$ such that $h_{\alpha}^{0}(\beta)=h(\beta)$ if $\beta<\alpha$ and $h_{\alpha}^{0}(\beta)=0$ of $\beta \geq \alpha$. Analogously $h_{\alpha}^{1}(\beta)=h(\beta)$ if $\beta<\alpha$ and $h_{\alpha}^{1}(\beta)=1$ if $\beta \geq \alpha$. The sets $A_{0}=\left\{h_{\alpha}^{0}: \alpha<\omega_{1}\right\}$ and $A_{1}=\left\{h_{\alpha}^{1}: \alpha<\omega_{1}\right\}$ are closed and disjoint in $G$ but cannot be separated by open sets in $G$.

Now we have only the $k$-property to deal with to finish our proof. Let $S=\left\{f \in 2^{\omega_{1}}|\{\alpha: f(\alpha) \neq 0\}|<\omega\right\}$ and $G=S \cup\left(S+f_{1}\right)$. It suffices to prove that $G$ is not a $k$-space.

The set $S$ is not closed in $G$. Take any compact $K \subset G$. Then $t(K) \leq \omega$ because $K$ is a countable union of its compact $F U$-subspaces. If $K \cap S$ is not closed then there is a countable $A \subset K$ with $\bar{A} \not \subset S$ but this is impossible.

We have proved everything we needed in 3.3 .

3.4. Remark. It seems that no classical properties are countably additive in topological groups. This is easily seen if the group $S$ from the above argument is considered. In fact, $S$ is $\sigma$-discrete, but has no properties from the list $M$ in 3.1 .

3.5. Questions. Is paracompactness finitely additive in topological groups? Is any of the properties in 3.3 finitely additive in locally convex spaces? 


\section{REFERENCES}

1. M. G. Tkačenko, Ob odnom svoistve bicompactov (On a property of compact spaces), Seminar po obshchei topologii (A seminar on general topology), Moscow State Univ. P.H., Moscow, 1981, pp. 149-156. (Russian)

2. __ Ob odnom rezul'tate E. Maikla $i$ M. E. Rudin (On a result of E. Michael and M. E. Rudin), Vestnik Moskov. Gos. Univ. Ser. Mat., 1981, no. 5, 47-50. (Russian)

3. E. G. Pytkeev and N. N. Yakovlev, On bicompacta, which are unions of spaces defined by means of coverings, Comment. Math. Univ. Carolin. 21 (1980), 247-261.

4. Some theorems on absolute neighbourhood retracts, Ark. Math. 1 (1951), 389-408.

5. V. I. Kuz'minov, Gomologicheskaya teoriya razmernosti (Homological dimension theory), Uspehi Mat. Nauk 23 (1968), no. 5, 3-49. (Russian)

6. A. V. Arhangel'skii, $O$ sootnosheniyah mezhdu invariantami topologicheskih gruppi ih podprostranstv (On relationship between cardinal functions on topological groups and their subspaces), Uspehi Mat. Nauk 35 (1980), no. 3, 3-22. (Russian)

7. A. V. Arhangel'sii and D. B. Šahmatov, $O$ potochechnoi approximatskii proizvol'nyh functsii schetnymi semeistvami nepreryvnyh functsii (On pointwise approximation of arbitrary functions by countable families of continuous functions), Trudy Sem. Petrovsk. 13 (1988), 206-227. (Russian)

Department of Mathematics, Moscow State University, Moscow 119899, Russia 